\title{
Acute cell viability and nitric oxide release in lateral menisci following closed-joint knee injury in a lapine model of post-traumatic osteoarthritis
}

\author{
Megan L Killian ${ }^{3,4}$, Roger C Haut ${ }^{2}$ and Tammy L Haut Donahue ${ }^{1,4^{*}}$
}

\begin{abstract}
Background: Traumatic impaction is known to cause acute cell death and macroscopic damage to cartilage and menisci in vitro. The purpose of this study was to investigate cell viability and macroscopic damage of the medial and lateral menisci using an in situ model of traumatic loading. Furthermore, the release of nitric oxide from meniscus, synovium, cartilage, and subchondral bone was also documented.

Methods: The left limbs of five rabbits were subjected to tibiofemoral impaction resulting in anterior cruciate ligament (ACL) rupture and meniscal damage. Meniscal tear morphology was assessed immediately after trauma and cell viability of the lateral and medial menisci was assessed 24 hrs post-injury. Nitric oxide (NO) released from joint tissues to the media was assayed at 12 and 24 hrs post injury.

Results: $\mathrm{ACL}$ and meniscal tearing resulted from the traumatic closed joint impact. A significant decrease in cell viability was observed in the lateral menisci following traumatic impaction compared to the medial menisci and control limbs. While NO release was greater in the impacted joints, this difference was not statistically significant.

Conclusion: This is the first study to investigate acute meniscal viability following an in situ traumatic loading event that results in rupture of the ACL. The change in cell viability of the lateral menisci may play a role in the advancement of joint degeneration following traumatic knee joint injury.
\end{abstract}

Keywords: Meniscus, Trauma, Knee, Cell viability, Anterior cruciate ligament

\section{Background}

Anterior cruciate ligament (ACL) injury is one of the most common injuries to the knee [1]. Concomitant injuries, such as meniscal tears and bone bruising, often occur with traumatic ACL rupture [1]. These injuries are known to play a role in soft tissue damage and the progression of post-traumatic osteoarthritis (PTOA) of the knee. Acute morphological and biological perturbations, such as cartilage fissuring, subchondral bone bruising, and chondrocyte death have been associated with rapid joint degeneration [2]. Such injuries, along with posterolateral meniscal tearing, have been observed after traumatic impaction that leads to ACL rupture $[3,4]$.

\footnotetext{
* Correspondence: Tammy.Donahue@ColoState.edu

'Department of Mechanical Engineering, Colorado State University, 1374

Campus Delivery, Fort Collins, CO, USA

${ }^{4}$ Department of Mechanical Engineering, Michigan Technological University, Houghton, MI, USA

Full list of author information is available at the end of the article
}

Traumatic impaction has been shown to induce acute cell death and macroscopic damage to cartilage and menisci in vitro [5,6]. Chondrocyte cell death following cartilage injury has been associated with increased expression of pro-inflammatory cytokines and increased release of nitric oxide (NO) in such studies. High levels of $\mathrm{NO}$ can lead to increased apoptosis in chondrocytes [7] and synoviocytes [8], and increased NO levels have been associated with the progression of osteoarthritis. Additionally, in vitro cyclic mechanical loading has been shown to play a role in NO release from joint tissues such as the meniscus [9] and cartilage [10]. Previous studies have looked at NO release in a lapine model following transection of the anterior cruciate ligament [11] and following partial meniscectomy [12]. These studies demonstrated an increase in $\mathrm{NO}$ production from meniscal tissue following prolonged ligament and meniscal injury, however these studies did not replicate PTOA via traumatic compressive 
loading, nor were the acute changes to the menisci documented. Understanding how the menisci behave in the acute phase following traumatic joint impaction may help guide future therapies following traumatic knee injury in order to prevent the development of PTOA.

The purpose of this study was to investigate tear morphology, cell viability in the medial and lateral menisci and investigate the release of $\mathrm{NO}$ from joint tissues following traumatic impaction of an in situ, closed-joint animal model. Specifically, it was hypothesized that traumatic impaction would result in decreased cell viability compared to control joint tissues. We expected increased cell death in the lateral menisci (LM) compared to medial menisci (MM) following traumatic impaction based on the higher risk of LM tearing following this type of joint impaction [4]. Additionally, it was hypothesized that the impacted joints would demonstrate a greater release of NO than control tissues.

\section{Methods}

\section{Traumatic impaction}

Five skeletally mature Flemish Giant rabbits $(5.9 \pm 0.8 \mathrm{~kg})$ were used in the study following approval by the Institutional Animal Care and Use Committee. The animals were housed in individual cages $\left(61 \times 122 \times 46 \mathrm{~cm}^{3}\right)$ prior to the study. Animals were tranquilized with $1 \mathrm{mg} / \mathrm{kg}$ acepromazine and anesthetized using 5\% isoflorane and 1\% oxygen. The animals were euthanized by overdose of isoflorane and intracardial injection of potassium chloride. Immediately after death, the animals received a blunt force insult to the left tibiofemoral (TF) joint using a previously described drop tower [4]. The drop tower sled was arrested electronically after one impact. A pre-crushed, deformable aluminum impact interface (Hexcel, Stamford, CT, 3.76 MPa crush strength) was used to ensure uniform loading over the femur. The interface was mounted in front of a $4.45 \mathrm{kN}(1000 \mathrm{lb})$ load transducer $\left(\mathrm{ICP}^{\circledR}\right.$ force sensor, model 208C04, PCB Electronics, Depew, New York, USA) (Figure 1). The impact mass was $1.75 \mathrm{~kg}$ and it was dropped from $0.88 \mathrm{~m}$. The animal was laid supine with the knee flexed at $90 \mathrm{deg}$. The foot was fixed in a boot with two Velcro straps. Two additional Velcro straps crossed over the femur. The left leg was positioned such that the dropped mass struck the distal femur, which resulted in a "kissing" impaction of the femur onto the posterior tibial plateau (Figure 1). The impact was such that load is transferred through the patella to the femur, and through the femur to the tibia. The patella was in line with the impact area and its imprint was always present in the deformable interface. No damage was noted to the patella in any of the impacted limbs. This impaction resulted in anterior tibial subluxation. After impaction, an anterior drawer test was used to diagnose potential ACL tears. Briefly, an examiner (MLK) held the tibia and pulled anteriorly and visually assessed translation of the tibia with respect to the femur. The right leg served as a non-impacted, internal control.

Immediately following impaction (within $\sim 1$ minute), both impacted and control limbs were disarticulated at the knee and dissected under sterile conditions. Knees were disarticulated and the presence and location of meniscal tears, as well as integrity of the ACL, was determined. The tibial plateau and femoral condyles were then harvested approximately $0.6-0.8 \mathrm{~cm}$ from the proximal and distal ends, respectively. Menisci remained attached to tibial plateaus. Once removed, tibiae $(\mathrm{T})$ and femur (F) blocks were rinsed in PBS and growth media (44\% Hams F12, 44\% Dulbecco's Modified Eagle Medium (DMEM), 10\% fetal bovine serum, and $2 \%$ penicillin/ streptomycin; $37^{\circ} \mathrm{C}$ ). Within 1 hour post-impaction, whole tibiae and femur blocks were submerged in individual wells containing $10 \mathrm{~mL}$ growth media and incubated at $37^{\circ} \mathrm{C}$ and $5 \% \mathrm{CO}_{2}$. Media was removed at $12 \mathrm{hrs}$ postharvest $\left(t_{12}\right)$ and replaced with fresh media. Media was again removed at $24 \mathrm{hrs}$ post-harvest $\left(t_{24}\right)$.

\section{Tibiofemoral contact pressures}

To maintain integrity of all structures, including the synovium and skin, prior to impaction, contact pressure data was collected for only one animal because it required an incision and opening of the joint prior to impaction. One animal (A5) was used to document contact pressures in the knee during impaction using a previously described protocol [13]. Packets of stacked, medium and low range, pressure sensitive film (Prescale, Fuji Film Ltd., Tokyo, Japan) were inserted through the joint, anterior to posterior between the femoral condyles and the menisci, to record contact pressure in the joint during impaction. After impact, the films were removed from the packet and scanned (Scanmaker MRAS-1200E6, Microtek, Taiwan). The medium range film was digitized (Photostyler v1.1A, Aldus Co., Seattle, WA). Total area of contact was calculated from markings on the pressure film. A custom MATLAB (Mathworks, Natick, MA) script was used to determine color intensity of pixels, ranging from white (no pressure) to red (high pressure), and calibrated in a material test system using known pressures. Pressure was averaged across the film and peak pressures were determined as well as the contact area in each tibial compartment having pressures over $20 \mathrm{MPa}$.

\section{Cell viability}

At $t_{24}$, menisci were removed from tibial plateaus and 2-3 parallel $150 \mu \mathrm{m}$ thick coronal sections from the central regions of the lateral (LM) and medial (MM) menisci were obtained. Maximal cell death is observed after $24 \mathrm{hr}$ of culture, and therefore this period of incubation was used [13]. If a meniscal tear was present, the slices included the 


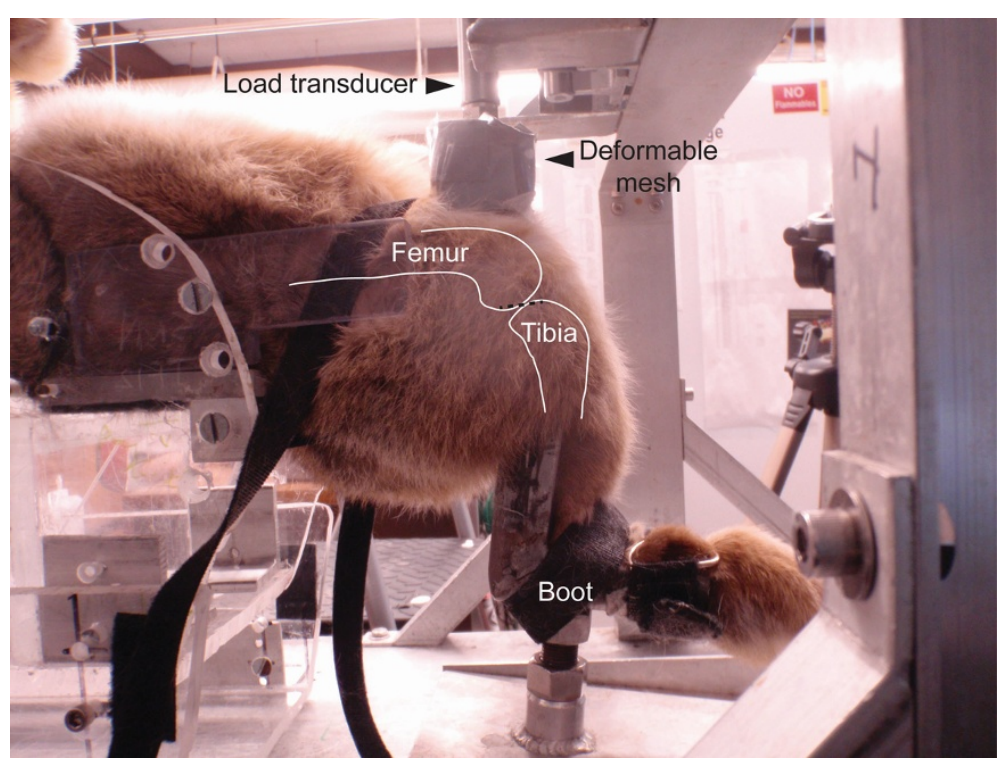

Figure 1 Impact experiments were performed by dropping a gravity-accelerated mass onto the flexed tibial-femoral joint. The rabbit knee was positioned such that the deformable head struck the distal femur in order to induce anterior tibial subluxation, ACL rupture and meniscal tearing.

tear, while remaining as centrally located as possible. Sections of menisci were immediately incubated in $2 \mathrm{mM}$ ethidium homodimer-1 and $4 \mathrm{mM}$ Calcein AM (Life Technologies, Grand Island, NY) in PBS and incubated for 30-45 min in the dark at room temperature. Images were captured for both live (green fluorescence) and dead (red fluorescence) cells using a fluorescence microscope (Olympus AX70 microscope and DP70 camera, Center Valley, PA) at the same magnification and exposure (10X). CellC was used to count live and dead cells from individual field of view images [14], where the percentage of cell viability was equal to the amount of live cells divided by the total sum of dead and live cells. Data from the 2-3 $150 \mu \mathrm{m}$-thick slices for each menisci were averaged for four of the five animals in the study.

\section{Nitric oxide release}

The release of $\mathrm{NO}$ into the tibial and femoral culture media at $t_{12}$ and $t_{24}$ was quantified using a commercially available assaying kit (Nitrate/Nitrite Colorimetric Assay Kit, Cayman Chemical Company, Ann Arbor, MI). Conditioned media were filtered using a $10 \mathrm{kD}$ cut-off filter (Millipore Microcon YM-10, Bedford, MA, USA) prior to performing nitrate/nitrite assays. The final concentration was normalized to wet weight ( $\mathrm{g}$ ) of the tissue $(\mathrm{N}=3$ for each time point).

\section{Statistics}

Statistical analysis was performed using Prism (Graphpad 6.0d). Wilcoxon-signed ranks tests were used to determine statistical differences in cell viability between control and impacted limbs for lateral menisci (LM) and medial menisci (MM). Descriptive statistics were used to compare $\mathrm{NO}$ release from control and impacted joint tissue after 12 and 24 hrs of incubation. P values $<0.05$ were used for all analyses.

\section{Results}

Meniscal tears and ACL rupture for each animal is described in Table 1. The impact force to induce ACL rupture and meniscal tearing was $737 \pm 96 \mathrm{~N}$ (mean $\pm \mathrm{SD}$ ). Four of the five animals experienced ACL damage, although all five were diagnosed with a characteristic "clicking" noise during anterior drawer tests prior to dissection. Control limbs did not demonstrate either meniscal or $\mathrm{ACL}$ tears at the time of dissection (Figure 2A). Following traumatic impaction, four of five animals experienced LM tears, primarily longitudinal or radial tears in the central-posterior region. One animal developed a LM tear without ACL rupture and two of the five animals had MM tears with concomitant ACL injury following traumatic impaction. One animal experienced both LM and MM tears with an ACL tear following impaction (Figure 2B). All meniscal tears were located in the central and/or posterior regions (Figure 2B) with two tears having full-width LM radial tears. Qualitative assessment of synovial tissue indicated that the parameniscal regions of the impacted tissue, following a $24 \mathrm{hr}$ period of incubation, indicated increased tissue swelling compared to control tissue (Figure 2C\&D).

Measurement of contact pressures in a single knee joint showed that the average contact pressure generated 
Table 1 Meniscal tears and ACL rupture descriptions

\begin{tabular}{lllll}
\hline Animal & Sex & Weight $\mathbf{( k g )}$ & Impact force $\mathbf{( N )}$ & Tear description \\
\hline 1 & M & 5.1 & 727 & ACL tear, medial and lateral meniscal tears \\
2 & M & 6.0 & 672 & NO ACL tear, but did have lateral posterior meniscal tear \\
3 & M & 5.4 & 890 & Partial ACL tear, lateral posterior meniscal tear \\
4 & 7.1 & 661 & ACL tear, medial meniscus tear in posterior region \\
5 & F & 5.8 & 733 & ACL tear, lateral meniscal tear \\
\hline
\end{tabular}

in the lateral and medial compartments of the knee were 27.5 MPa and 22.7 MPa, respectively. The peak contact pressures in the lateral and medial compartments were $50.9 \mathrm{MPa}$ and 48.4 $\mathrm{MPa}$, respectively. The contact area with pressures over $20 \mathrm{MPa}$ were $19.7 \mathrm{~mm}^{2}$ on the lateral plateau and $18.5 \mathrm{~mm}^{2}$ on the medial plateau.

From a qualitative standpoint, uninjured controls demonstrated consistent and uniform cell viability across meniscal sections, with some dead cells evenly distributed across the sections (Figure 3A\&B). In menisci from impacted limbs, cell death was most apparent along the torn edges (Figure $3 \mathrm{C}$ ). Impaction led to a strong statistical trending decrease $(\mathrm{p}=0.06)$ in cell viability in the LM compared to the same menisci of controls (Figure 3D). Changes in cell viability of the MM were not statistically different between control and impacted limbs (Figure 3E).

Cumulative NO following traumatic impaction was highly variable $(162 \pm 104 \mu \mathrm{M} /$ weight $[\mathrm{g}]$ femur, $64 \%$ confidence interval (CI); $169 \pm 126 \mu \mathrm{M} /$ weight $[\mathrm{g}] /$ tibiae $74 \%$ CI) compared to control limbs $(110 \pm 30 \mu \mathrm{M} /$ weight $[\mathrm{g}]$ femur, $27 \% \mathrm{CI} ; 112 \pm 47 \mu \mathrm{M} /$ weight $[\mathrm{g}]$ tibiae, $42 \% \mathrm{CI}$ ) after 24 hrs (Figure 4). Similar results were present in the $t_{12}$ samples from the tibia and femur. Variability in NO release was $64-83 \% \mathrm{CI}$ for impacted joints and $14-42 \% \mathrm{CI}$ for control joints.

\section{Discussion}

This is the first study to explore the role of in situ loading on meniscal cell viability, specifically involving traumatic impaction, ACL rupture, and acute meniscal tearing. Prior to the current study, viability of meniscal cells following traumatic loading has only been explored in vitro using explants and tissue culture in a limited number of studies $[6,15]$. While studying impaction in vitro has allowed researchers to isolate the tissue and cell populations of interest from external biological factors (e. g. synoviocytes, macrophages), it is likely that tissue culture and explant loading could alter the boundary conditions and mechanical behavior of tissues compared to that experienced in the in situ environment [5].

In the present study, meniscal cell death was largely localized near and along the surfaces of tears. Similar
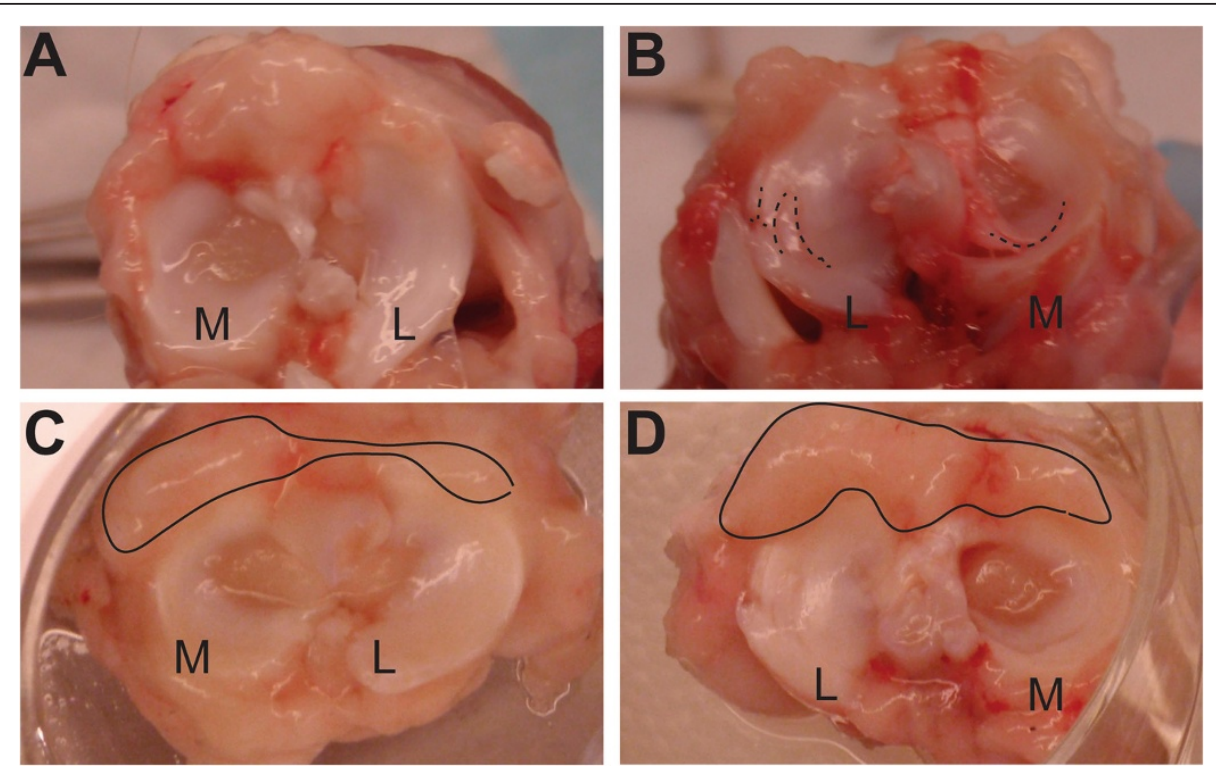

Figure 2 Gross morphology of menisci showed tears and synovial swelling at $\mathbf{2 4 h r}$ post-impaction. Gross morphology of menisci immediately after dissection in (A) control knee and (B) impacted left knee resulting in ACL tear. Dashed lines highlight tears in the posterior region of both menisci. Synovial swelling after 24 hrs incubation was generally greater for (D) impacted knees compared to (C) control limbs. $\mathrm{L}=$ lateral, $\mathrm{M}=$ medial. 

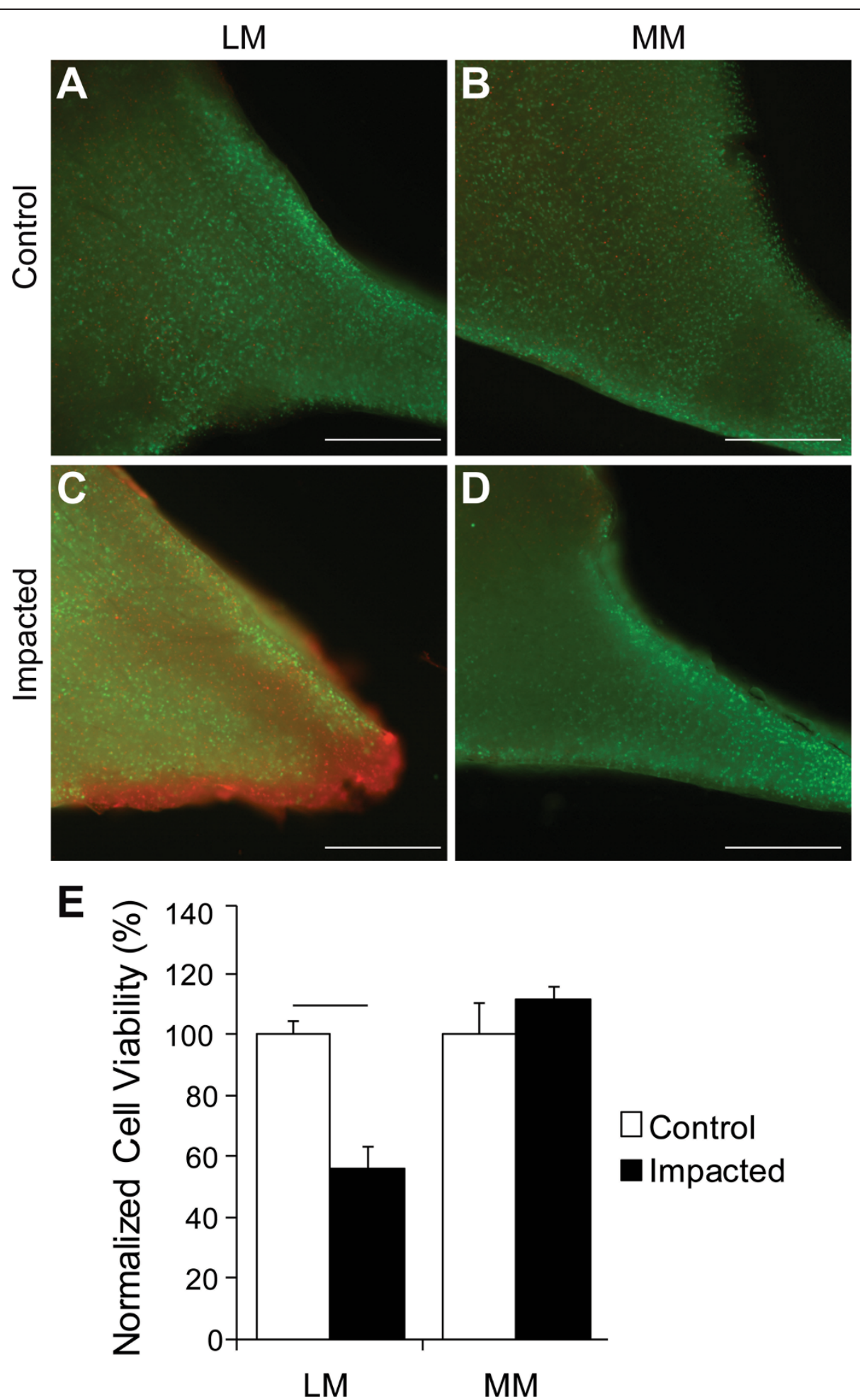

Figure 3 Cell viability was reduced at meniscal tear sites. A-D) Representative cell viability images of LM (A \& C) and MM (B \& D) from control (top panel) and impacted (middle panel) knees. Increased cell death (red cells) was appreciable at tear edge of the impacted LM. E) Cell viability of impacted limbs was significantly reduced in LM with impaction compared to control. Data is shown as percentage of cell viability of control or impacted limbs normalized to the percentage of cell viability of control limbs. Bar indicates a strong statistical trending decrease in cell viability $(p=0.06)$. Scale bar $=500 \mu \mathrm{m}$. 


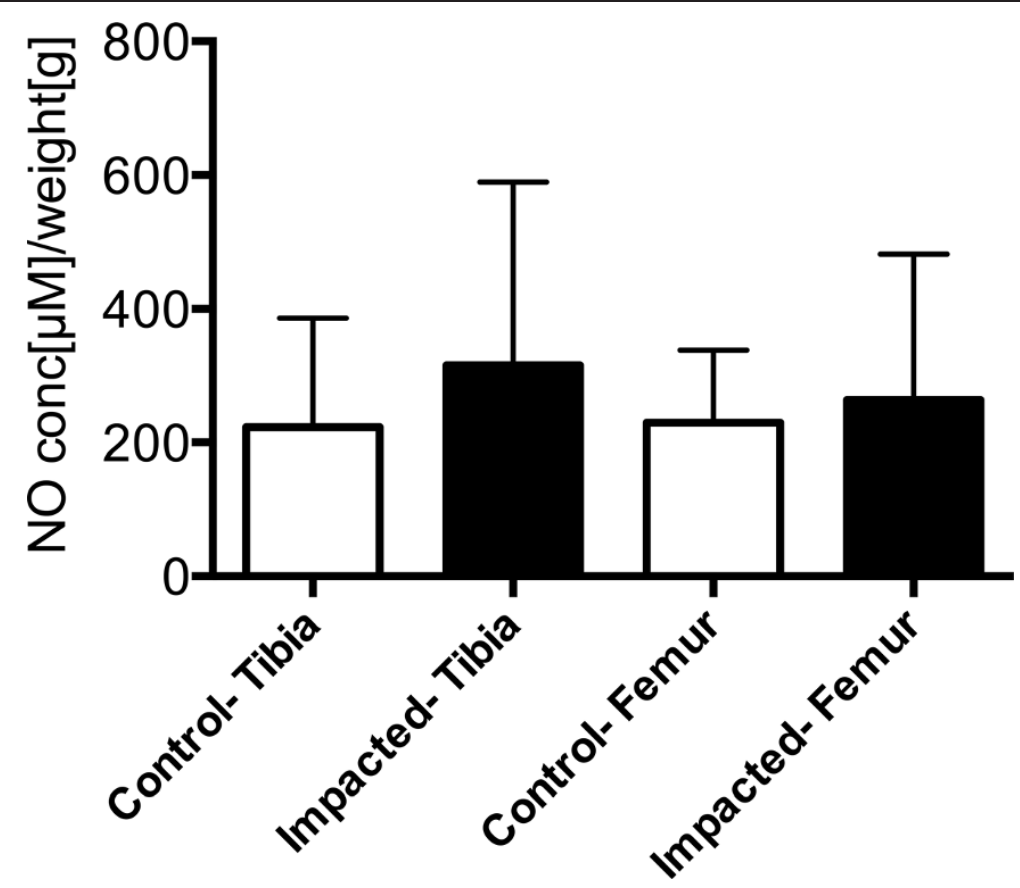

Figure 4 Cumulative NO release of meniscotibial (T) and femoral (F) tissue from control and impacted limbs measured in the culture media in the first $24 \mathrm{hrs}$ after impaction. NO concentration $(\mu \mathrm{M})$ was normalized to wet weight of tissue (g). Mean $\pm 95 \%$ confidence intervals.

findings have been observed following in vitro studies on meniscal and cartilage explants [5,6]. In the current study, cell death was most apparent in the LM, and such decreases in cell viability may be explained by the increased presence of meniscal tears in that compartment in conjunction with a suggested increase in contact pressures at the time of impaction. A decrease in cell viability may suggest the need for acute pharmacological intervention following traumatic injury. For example, treatment with cell surfactants, such as poloxamer-P188 (P188), a non-ionic surfactant, has previously demonstrated success maintaining cell viability in chondrocytes following impact loading of articular cartilage [16-19]. Such treatments may improve cell membrane stiffness and their ability to induce cellular and matrix repair following a single high-impact mechanical load [19]. At present, it is unclear whether an acute decrease in cell viability at the time of injury impairs the remodeling and healing of the injured meniscus following a traumatic impaction. Additionally, it is unknown whether meniscal cells play a role in degenerative and/or healing adaptations in the chronic setting following traumatic meniscal/ACL injury. Future work is needed to investigate potential molecular cues directing cellular activity and autophagy initiated by meniscal fibrochondrocytes.

Recently, in vivo studies have established the role of high-stress loading on cartilage viability of the knee using the lapine animal model [13]. Isaac et. al., measured cell death of cartilage in vivo using an impact protocol similar to the current study, without tibial translation in order to prevent anterior tibial subluxation. This constraint prevented ACL rupture and meniscal tears [13]. In their study, elevated chondrocyte death was observed in lateral more than the medial compartment of the knee. Their findings suggested that increased chondrocyte death in the lateral compartment was due primarily to the relatively higher levels of impact-induced contact stresses generated in the lateral versus in the medial compartment of the lapine knee, as reflected in the current study. Localization of stress and regions of high cell death are then likely correlated and dependent on the mechanism of energy transfer across the joint during traumatic loading. While it is generally understood that the medial compartment observes higher contact loads than the lateral compartment during normal loading in the human knee [20,21], localized stresses on the articulating surfaces of the knee during traumatic loading conditions have only been marginally characterized in translatable animal models [22]. In a recent cadaveric model of ACL injury patterns, Levine et al. demonstrated that valgus rotation of the tibia prior to impact loading resulted in concomitant lateral meniscal tears in conjunction with ACL rupture [23]. Their study suggested the dependence of tibial plateau injury, but not ACL rupture, on applied loading conditions [23]. Additionally, while their study demonstrated orientation-driven injuries to the lateral meniscus, post-impaction injuries to the medial menisci were not apparent. In the present study, we demonstrate that deep-knee flexion with high impact loading on the 
tibiofemoral joint leads to predominantly posterolateral meniscal injuries, with and without ACL rupture, in a novel lapine model of PTOA. Future studies should focus on localized loading and contact stresses of the menisci, ACL, and tibial plateau at the time of traumatic impaction using this animal model in order to gain insight into realistic and translatable models of sportsrelated knee injuries.

Traumatic injury to the knee following events such as sport jumping and landing (e.g. skiing or basketball) has been shown to lead to increased axial tibia loading, and such loading can lead to increased anterior tibial forces, anterior tibial translations and internal tibial rotation [24]. In the present study, joint injury was induced by anterior tibial subluxation, which primarily led to longitudinal tears in the posterior aspect of the lateral and/or medial menisci and ACL ruptures. Anterior tibial translation and subsequent rupture of the ACL following impaction may have encouraged a combined "pinched" shearing of the menisci along with compression of the femur on the posterior edge of the tibial plateau. We speculate that the presence of meniscal and ACL tears were initiated by soft tissue compression and accumulated strain energy in these tissues which would have otherwise been attenuated through intact underlying soft tissue and bone.

Nitric oxide is a known byproduct of chondrocytes in the presence of inflammatory markers, and increased levels of NO can lead to increased chondrocyte death. Therefore, we investigated the release of NO from cartilage and subchondral bone of the femur as well as cartilage, subchondral bone and menisci from the tibia. In this study, we did not observe a statistically significant increase in NO release following traumatic impaction in the acute setting. The variability in the NO data, as indicated by high confidence variables, suggests our data are inconclusive. It is possible that there could be no difference between control and impacted limbs; however, with approximately 70\% CI for impacted limbs, it is not possible to draw strong conclusions from this study. Additionally, it is also possible that acute traumatic impaction does not lead to release of NO from the meniscus or articular cartilage within 24 hours of injury, or that the en-bloc samples of cartilage, meniscus, and bone incubated together in culture media had an overwhelming effect on the detection of NO released to the media. Long term adaptations following transection of the ACL with meniscal injury may further increase NO production, as shown in other studies [11,12]. It is possible that we did not select a longitudinal time point with sufficient duration to detect changes between control and impacted limbs. Nonetheless, data from the present study representing findings in NO release should be taken with caution.

This study is not without limitations. One limitation was the variability of ACL and meniscal injury in our sample population. It is likely that anatomical differences between animals led to differences in post-trauma outcomes in ligament and meniscal injuries. Specifically, the rabbits utilized in this study were not identical in size and weight; differences in bone architecture, fatty deposition, and muscle mass may have played a role in the degree of injury incurred following impaction. These variables were not accounted for in our study design.

Another limitation was the lack of separation between the different articular tissues in post-dissection culture. It is unclear, from the experiments performed in this study, which tissues were responsible for the release of $\mathrm{NO}$ and to what magnitude each individual tissue contributed. Therefore, these findings are only suggestive and do not qualify menisci, cartilage, subchondral bone, or synovium as primary donor(s) of NO following traumatic joint injury. This study merely provides a first-pass as to how the joint, as a collection of tissues, contributes to the release of NO. Future studies should isolate and culture individual tissues in order to better assess the isolated contributions of these tissues in NO release. Additionally, this study is limited by the lack of external controls in assessing meniscal cell viability with and without impaction. This study utilized contralateral limbs of impacted knees to assess cell viability and NO release. While paired comparisons provide increased statistical power, it is possible that genetic variability of the lapine population selected may have biased our findings. Lastly, this study is limited by its utilization of a lapine model to induce traumatic ACL rupture, and may not be directly translatable to the human patient. Specifically, it is not clear from this work whether our findings in a lapine model can be translated to the human case. Specifically, the joint anatomy of the lapine knee is not identical to that of the human [25], and these differences may play a role in differential joint loading biomechanics between the two species.

This study illustrated that meniscal defects associated with impaction-induced ACL rupture led to fibrochondrocyte cell death primarily localized near surface lesions in the lateral menisci within the first $24 \mathrm{hrs}$ following injury. An understanding of the cellular response of meniscal tissue to traumatic impaction may lead to alterations in acute treatments that could potentially help to delay progression of meniscal degradation and development of knee joint PTOA.

\section{Conclusion}

Traumatic impaction, resulting in meniscal tears, led to acute cell death localized to surface lesions in a rabbit model of post-traumatic OA.

Competing interests

The authors declare that they have no competing interests. 


\section{Authors' contributions}

MK carried out the experiments, cell viability tests, nitric oxide assay, data and statistical analysis and drafted the manuscript. THD and RH conceived the study, and participated in its design and coordination and helped to draft the manuscript. All authors read and approved the final manuscript.

\section{Acknowledgements}

This research was supported by the National Institutes of Health R21AR060464.

\section{Author details}

'Department of Mechanical Engineering, Colorado State University, 1374 Campus Delivery, Fort Collins, CO, USA. ${ }^{2}$ Orthopaedic Biomechanics Laboratories, Michigan State University, East Lansing, MI, USA. ${ }^{3}$ Department of Orthopaedic Surgery, Washington University School of Medicine, St. Louis, MO, USA. ${ }^{4}$ Department of Mechanical Engineering, Michigan Technological University, Houghton, MI, USA.

Received: 15 August 2013 Accepted: 27 August 2014

Published: 6 September 2014

\section{References}

1. Frobell RB, Le Graverand MP, Buck R, Roos EM, Roos HP, Tamez-Pena Totterman S, Lohmander LS: The acutely ACL injured knee assessed by MRI: changes in joint fluid, bone marrow lesions, and cartilage during the first year. Osteoarthr Cartil 2009, 17:161-167.

2. Johnson DL, Urban WP, Caborn DN, Vanarthos WJ, Carlson CS: Articular cartilage changes seen with magnetic resonance imaging-detected bone bruises associated with acute anterior cruciate ligament rupture. Am J Sports Med 1998, 26:409-414.

3. Isaac DI, Meyer EG, Haut RC: Development of a traumatic anterior cruciate ligament and meniscal rupture model with a pilot in vivo study. J Biomech Eng 2010, 132:064501.

4. Killian ML, Isaac DI, Haut RC, Déjardin LM, Leetun D, Haut Donahue TL: Traumatic anterior cruciate ligament tear and its implications on meniscal degradation: a preliminary novel lapine osteoarthritis model. J Surg Res 2010, 164:234-241.

5. Ewers BJ, Dvoracek-Driksna D, Orth MW, Haut RC: The extent of matrix damage and chondrocyte death in mechanically traumatized articular cartilage explants depends on rate of loading. J Orthop Res 2001, 19:779-784.

6. Kisiday JD, Vanderploeg EJ, Mcllwraith CW, Grodzinsky AJ, Frisbie DD: Mechanical injury of explants from the articulating surface of the inner meniscus. Arch Biochem Biophys 2010, 494:138-144.

7. Hashimoto S, Takahashi K, Amiel D, Coutts RD, Lotz M: Chondrocyte apoptosis and nitric oxide production during experimentally induced osteoarthritis. Arthritis Rheum 1998, 41:1266-1274.

8. Cillero-Pastor B, Martin MA, Arenas J, López-Armada MJ, Blanco FJ: Effect of nitric oxide on mitochondrial activity of human synovial cells. BMC Musculoskelet Disord 2011, 12:42.

9. Gupta T, Zielinska B, McHenry J, Kadmiel M, Haut Donahue TL: IL-1 and iNOS gene expression and NO synthesis in the superior region of meniscal explants are dependent on the magnitude of compressive strains. Osteoarthr Cartil 2008, 16:1213-1219.

10. Fermor B, Weinberg JB, Pisetsky DS, Misukonis MA, Banes AJ, Guilak F: The effects of static and intermittent compression on nitric oxide production in articular cartilage explants. J Orthop Res 2001, 19:729-737.

11. Hashimoto S, Takahashi K, Ochs RL, Coutts RD, Amiel D, Lotz M: Nitric oxide production and apoptosis in cells of the meniscus during experimental osteoarthritis. Arthritis Rheum 1999, 42:2123-2131.

12. Kobayashi K, Mishima H, Harwood F, Hashimoto S, Toyoguchi T, Goomer R Moriya $H$, Amiel D: The suppressive effect of hyaluronan on nitric oxide production and cell apoptosis in the central region of meniscus following partial meniscectomy. lowa Orthop J 2002, 22:39-41.

13. Isaac DI, Meyer EG, Haut RC: Chondrocyte damage and contact pressures following impact on the rabbit tibiofemoral joint. $J$ Biomech Eng 2008, 130:41018

14. Selinummi J, Seppälä J, Yli-Harja O, Puhakka JA: Software for quantification of labeled bacteria from digital microscope images by automated image analysis. Biotechniques 2005, 39:859-863.
15. Hufeland M, Schünke M, Grodzinsky AJ, Imgenberg J, Kurz B: Response of mature meniscal tissue to a single injurious compression and interleukin-1 in vitro. Osteoarthritis Cartilage 2013, 21(1):209-216.

16. Natoli RM, Athanasiou KA: P188 reduces cell death and IGF-I reduces GAG release following single-impact loading of articular cartilage. J Biomech Eng 2008, 130:41012.

17. Serbest G, Horwitz J, Jost M, Barbee K: Mechanisms of cell death and neuroprotection by poloxamer 188 after mechanical trauma. FASEB J 2006, 20:308-310.

18. Phillips DM, Haut RC: The use of a non-ionic surfactant (P188) to save chondrocytes from necrosis following impact loading of chondral explants. J Orthop Res 2004, 22:1135-1142.

19. Isaac DI, Golenberg N, Haut RC: Acute repair of chondrocytes in the rabbit tibiofemoral joint following blunt impact using P188 surfactant and a preliminary investigation of its long-term efficacy. J Orthop Res 2010 28:553-558

20. Zhao D, Banks SA, D'Lima DD, Colwell CW, Fregly BJ: In vivo medial and lateral tibial loads during dynamic and high flexion activities. J Orthop Res 2007, 25:593-602.

21. Mundermann A, Dyrby CO, D'Lima DD, Colwell CW Jr, Andriacchi TP: In vivo knee loading characteristics during activities of daily living as measured by an instrumented total knee replacement. J Orthop Res 2008, 26:1167-1172

22. Isaac DI, Meyer EG, Kopke KS, Haut RC: Chronic changes in the rabbit tibial plateau following blunt trauma to the tibiofemoral joint. J Biomech 2010, 43:1682-1688.

23. Levine JW, Kiapour AM, Quatman CE, Wordeman SC, Goel VK, Hewett TE, Demetropoulos CK: Clinically relevant injury patterns after an anterior cruciate ligament injury provide insight into injury mechanisms. Am J Sports Med 2013, 41(2):385-395.

24. Yeow C-H, Kong C-Y, Lee PV-S, Goh JC-H: Correlation of axial impact forces with knee joint forces and kinematics during simulated ski-landing. J Sports Sci 2011, 29:1143-1151.

25. Proffen BL, MCElfresh M, Fleming BC, Murray MM: A comparative anatomical study of the human knee and six animal species. Knee 2011, 19:493-499

doi:10.1186/1471-2474-15-297

Cite this article as: Killian et al:: Acute cell viability and nitric oxide release in lateral menisci following closed-joint knee injury in a lapine model of post-traumatic osteoarthritis. BMC Musculoskeletal Disorders 2014 15:297.

\section{Submit your next manuscript to BioMed Central and take full advantage of:}

- Convenient online submission

- Thorough peer review

- No space constraints or color figure charges

- Immediate publication on acceptance

- Inclusion in PubMed, CAS, Scopus and Google Scholar

- Research which is freely available for redistribution
C Biomed Central 